\title{
Erratum to: Gravity currents in two-layer stratified media
}

\author{
A. W. Tan - D. S. Nobes - B. A. Fleck - M. R. Flynn
}

Published online: 20 January 2011

(C) Springer Science+Business Media B.V. 2011

\section{Erratum to: Environ Fluid Mech DOI 10.1007/s10652-010-9174-z}

This brief communication corrects a minor error in the presentation of the model results described in $\$ 2$ of Tan et al. [1] (referred to hereafter as T.al.). Previously, we predicted that the solution of Eqs. 2.10 and 2.11 becomes unphysical for $g_{12}^{\prime} / g_{02}^{\prime} \geq 3 / 4$ irrespective of $h_{1}^{\prime}$. Upon closer inspection, we observe that model breakdown occurs at $g_{12}^{\prime} / g_{02}^{\prime}=3 / 4$ when $h_{1}^{\prime} \lesssim 0.50$ but extends beyond this value when $h_{1}^{\prime} \gtrsim 0.50$. This is illustrated in Fig. $2 \mathrm{a}$ which shows the surface plot of Fr vs. $g_{12}^{\prime} / g_{02}^{\prime}$ and $h_{1}^{\prime}$ and is identical to Fig. 3a of T. al. with the exception of the aforementioned correction. In like fashion, Figs. 1, 2, 3 and 4 are, respectively, the corrected versions of Figs. 2, 3, 4 and 5 from T. al. (Note, that the geometric variables $h_{1}^{\prime}, h_{1}, h_{2}^{\prime}$ and $h_{2}$ used here have been non-dimensionalized by $H$, the channel depth.) As these new figures make clear, the region of parameter space where the model is valid and, by extension, where the gravity current is predicted to be supercritical, is larger than described in the original manuscript. Notwithstanding this correction, the principal conclusions of T. al. remain unchanged.

In addition to calculating head loss along streamlines as we do with Eqs. 2.14 and 2.15 of T. al., one can also evaluate the global dissipation by determining the change of $D=$ $\int u\left(p+\frac{1}{2} \rho u^{2}+\rho g z\right) \mathrm{dz}$ from far upstream to far downstream. The expression for $\Delta D$, written in non-dimensional form reads

$$
\Delta D=\operatorname{Fr}\left[\frac{g_{12}^{\prime}}{g_{02}^{\prime}}\left(h_{2}^{\prime} h_{1}-h_{1}^{\prime} h_{2}^{\prime}\right)+\frac{g_{01}^{\prime}}{g_{02}^{\prime}} h_{1}^{\prime} h_{0}+h_{2}^{\prime} h_{0}-\frac{1}{2} \operatorname{Fr}^{2}\left(\frac{h_{1}^{\prime 3}}{h_{1}^{2}}+\frac{h_{2}^{\prime 3}}{h_{2}^{2}}\right)\right]
$$

The online version of the original article can be found under doi:10.1007/s10652-010-9174-z.

A. W. Tan · D. S. Nobes · B. A. Fleck · M. R. Flynn $(\varangle)$

Department of Mechanical Engineering, University of Alberta, Edmonton, AB T6G 2G8, Canada e-mail: mrflynn@ualberta.ca 

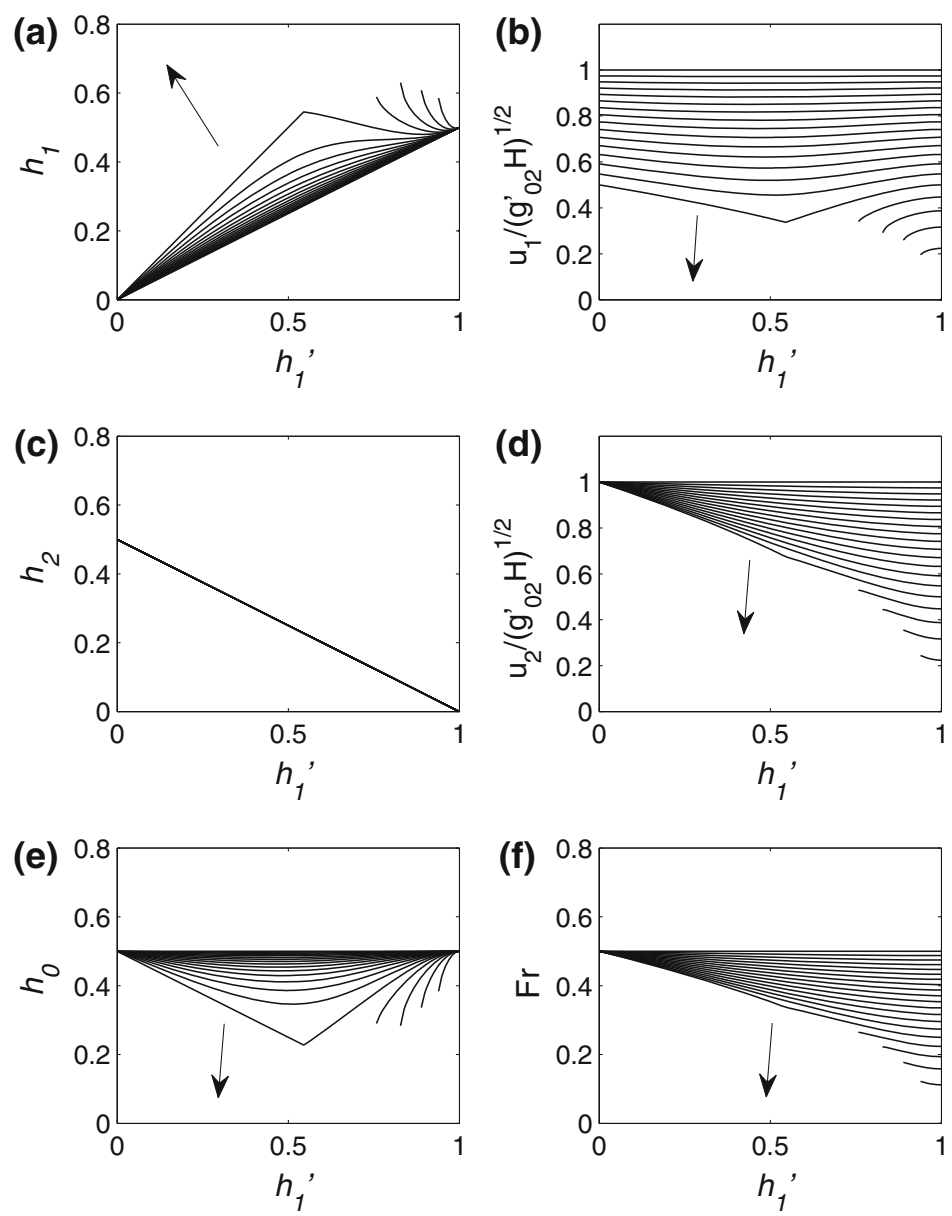

Fig. 1 As in Fig. 2 of T. al. except that the region of model validity is extended past $g_{12}^{\prime} / g_{02}^{\prime}=3 / 4$ when $h_{1}^{\prime} \gtrsim 0.5$. Broken lines for large $h_{1}^{\prime}$ and $g_{12}^{\prime} / g_{02}^{\prime}$ indicate model breakdown

[2]. Figure 5 shows $\Delta D$ vs. $g_{12}^{\prime} / g_{02}^{\prime}$ and $h_{1}^{\prime}$ using results of Eqs. 2.10 (panel a) and 2.11 (panel b). From Fig. 5a, we note that $\Delta D$ attains its maximum value when $h_{1}^{\prime} \simeq 0.49$ and $g_{12}^{\prime} / g_{02}^{\prime} \simeq 0.50$ while from Fig. $5 \mathrm{~b}, \Delta D$ attains its maximum value when $h_{1}^{\prime} \simeq 0.58$ and $g_{12}^{\prime} / g_{02}^{\prime} \simeq 0.43$. Although the location of this maximum varies slightly depending on the model assumptions, the surface plots of Fig. 5a, b are otherwise very similar. In particular, $\Delta D \rightarrow 0$ when $h_{1}^{\prime} \rightarrow 0, h_{1}^{\prime} \rightarrow 1$ or $g_{12}^{\prime} / g_{02}^{\prime} \rightarrow 0$. Also, and consistent with Figs. $2 \mathrm{~b}$ and $4 \mathrm{~b}, \Delta D \rightarrow 0$ when $g_{12}^{\prime} / g_{02}^{\prime}=3 / 4$ with $h_{1}^{\prime} \lesssim 0.5$. 

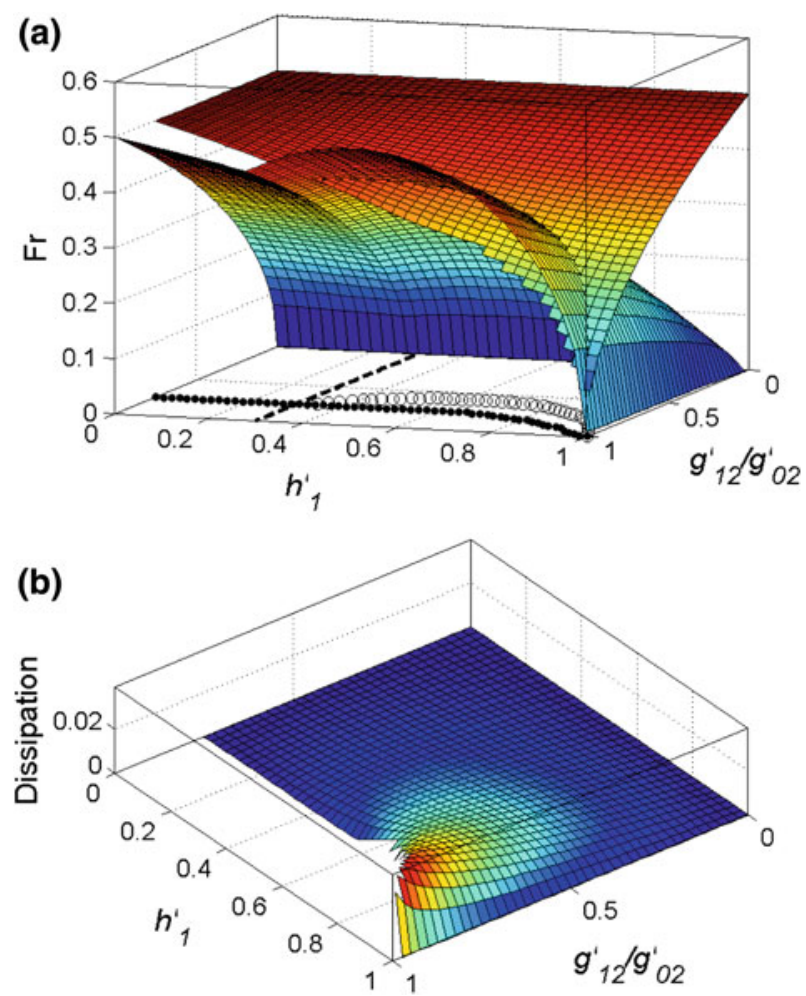

Fig. 2 As in Fig. 3 of T. al. except that the region of model validity is extended past $g_{12}^{\prime} / g_{02}^{\prime}=3 / 4$ when $h_{1}^{\prime} \gtrsim 0.5$ 

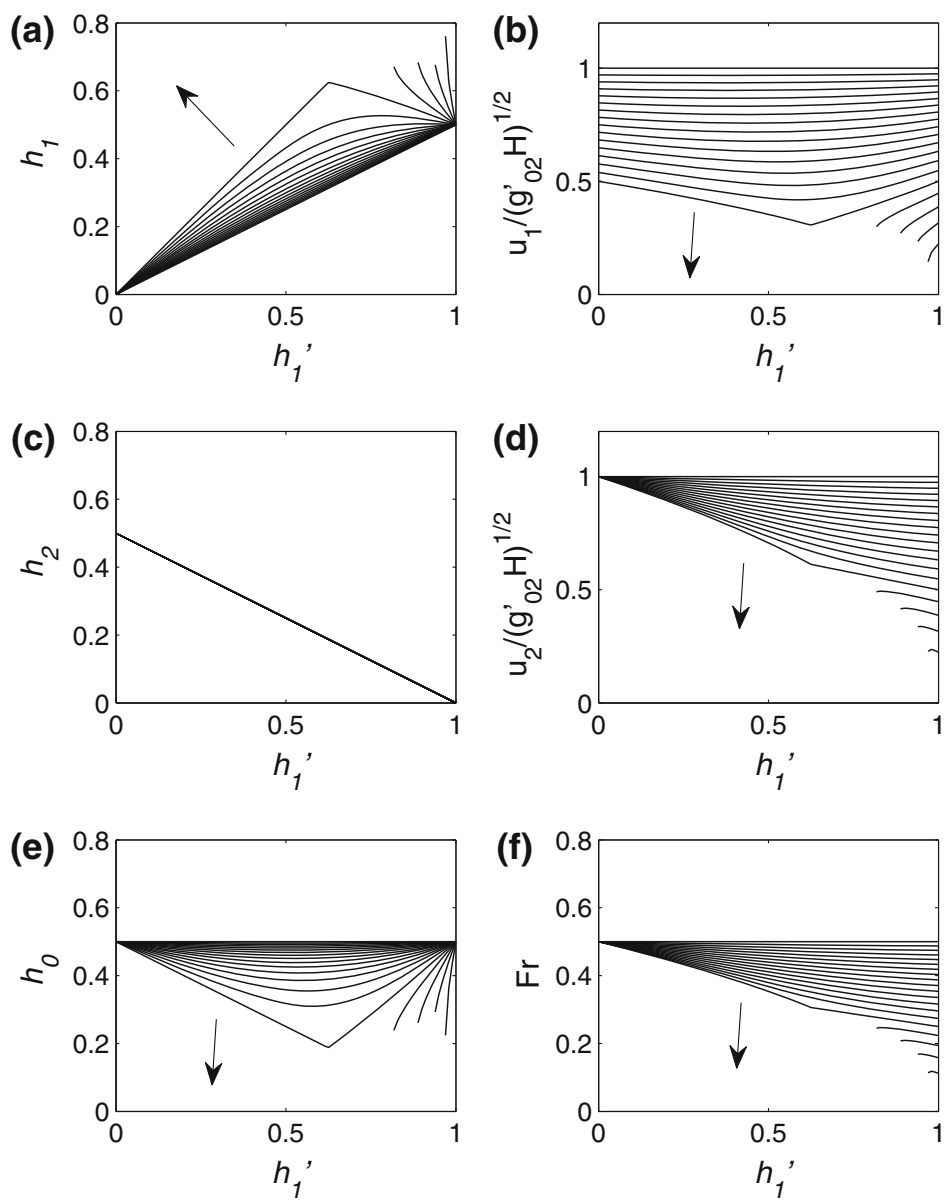

Fig. 3 As in Fig. 4 of T. al. except that the region of model validity is extended past $g_{12}^{\prime} / g_{02}^{\prime}=3 / 4$ when $h_{1}^{\prime} \gtrsim 0.5$. Broken lines for large $h_{1}^{\prime}$ and $g_{12}^{\prime} / g_{02}^{\prime}$ indicate model breakdown 

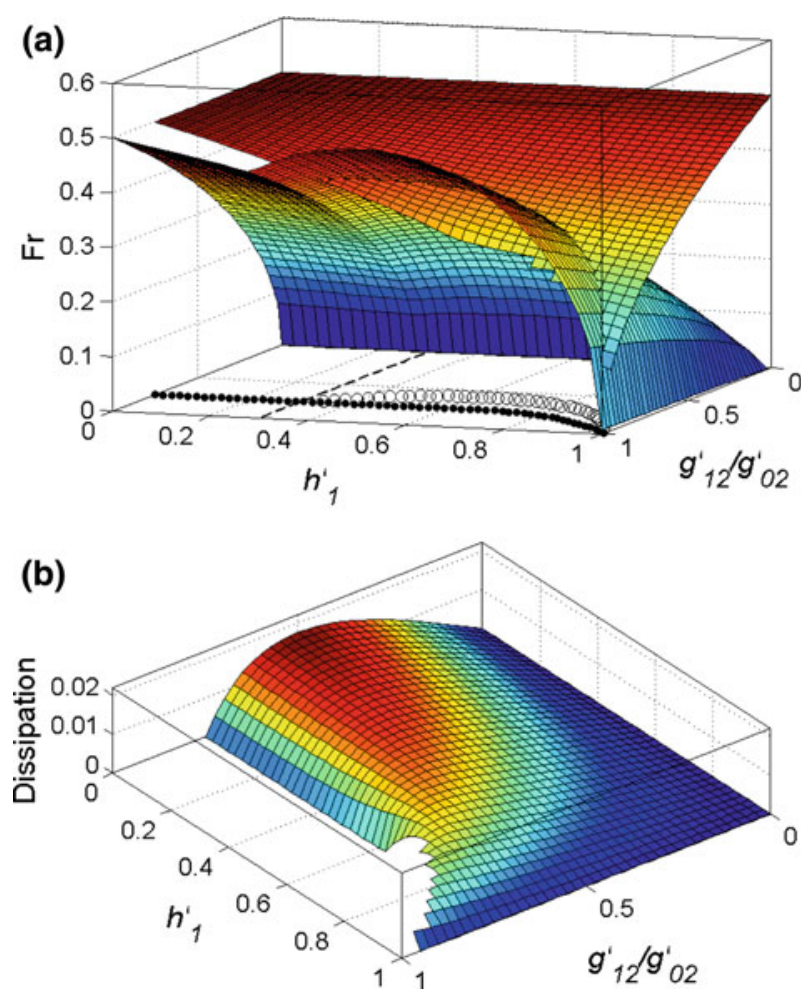

Fig. 4 As in Fig. 5 of T. al. except that the region of model validity is extended past $g_{12}^{\prime} / g_{02}^{\prime}=3 / 4$ when $h_{1}^{\prime} \gtrsim 0.5$ 
Fig. 5 The non-dimensional dissipation function, $\Delta D$, defined by (1), plotted against $h_{1}^{\prime}$ and $g_{12}^{\prime} / g_{02}^{\prime}$. Panels a and $\mathbf{b}$ corresponds to the dissipation associated with Eqs. 2.10 and 2.11 of T. al., respectively
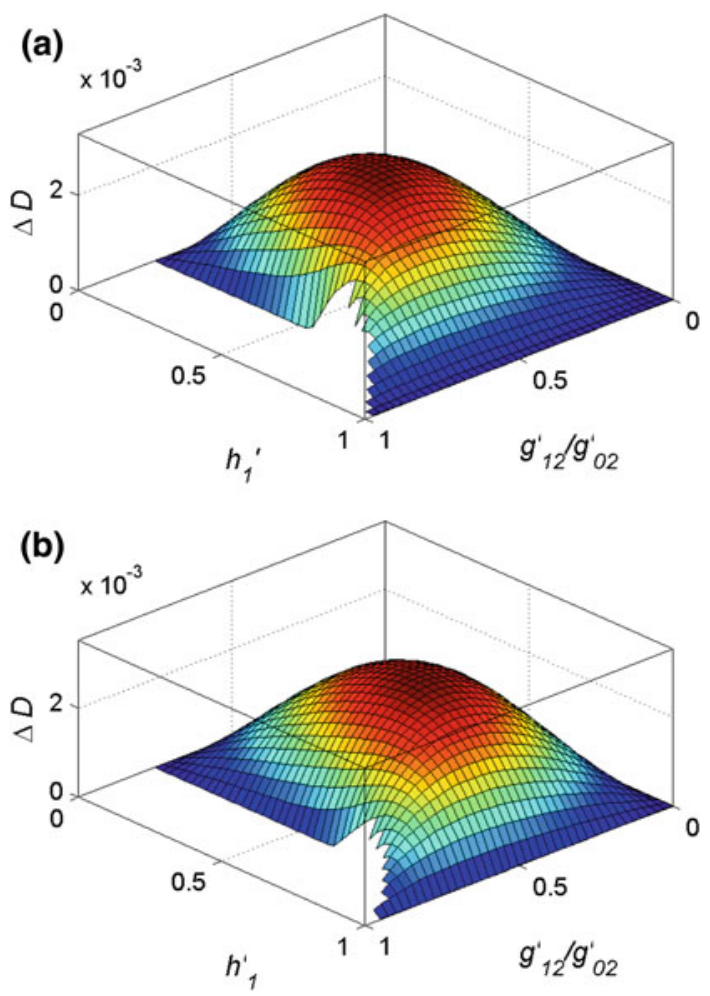

\section{References}

1. Tan A, Nobes D, Fleck B, Flynn M (2010) Gravity currents in two-layer stratified media. Environ Fluid Mech 1-21. doi:10.1007/s10652-010-9174-z

2. Ungarish M (2009) An introduction to gravity currents and intrusions. CRC Press, Boca Raton 